\title{
Homocysteine and Folate in Inflammatory Bowel Disease: Can Reducing Sulfur Reduce Suffering?
}

\author{
Annalisa Chiocchetti ${ }^{1} \cdot$ Flavia Prodam $^{1} \cdot$ Umberto Dianzani $^{1}$
}

Published online: 6 September 2018

○) Springer Science+Business Media, LLC, part of Springer Nature 2018

Sulfur-containing amino acids are essential to adequate mammalian nutrition. Amino acids are naturally occurring compounds involved in all biological processes, including metabolism, growth, and immunity; altered dietary intake or metabolic derangements affecting amino acids underlie the pathogenesis of multiple diseases.

Homocysteine is a sulfur-containing amino acid derived from the demethylation of methionine, an essential amino acid, which is abundant in animal proteins. Homocysteine metabolism is at the intersection of two metabolic pathways: remethylation and transsulfuration (Fig. 1) producing methionine and cysteine, respectively. In remethylation, homocysteine acquires a methyl group to form methionine in multiple reactions that are vitamin B9 (folate)- and B12 (cobalamin)-dependent. Subsequently, methionine is converted to form $S$-adenosylmethionine (SAM), a universal methyl donor to numerous nucleic acids, neurotransmitters, phospholipids, and hormones. Alternatively, in the transsulfuration pathway, homocysteine condenses with serine to form cystathionine and then cysteine, a vitamin B6 (pyridoxine)-dependent reaction. Thus, two enzymes and three water-soluble vitamins control serum homocysteine concentrations. Furthermore, homocysteine can also cyclize to form homocysteine thiolactone (HTL), a thioester considered to be a toxic intermediate due to an error-editing reaction in protein biosynthesis $[1,2]$.

The normal range of serum homocysteine concentration is $5-15 \mu \mathrm{mol} / \mathrm{L}$, influenced by many factors including gender and age. Blood homocysteine levels $>15 \mu \mathrm{mol} / \mathrm{L}$ are present in hyperhomocysteinemia that can be moderate $(16-100 \mu \mathrm{mol} / \mathrm{L})$ or severe $(>100 \mu \mathrm{mol} / \mathrm{L})$. Moderate hyperhomocysteinemia may be caused by polymorphisms

Umberto Dianzani

umberto.dianzani@med.uniupo.it

1 Department of Health Sciences, Interdisciplinary Research Center of Autoimmune Diseases, University of Piemonte Orientale, 28100 Novara, Italy in the genes regulating methionine metabolism, consuming a methionine-enriched diet, or by reduced vitamin B intake, particularly vitamin B12, a vitamin present only in food of animal origin that needs gastric intrinsic factor to be absorbed. Severe hyperhomocysteinemia may result from deficiencies in the enzymes metabolizing homocysteine through the demethylation and transsulfuration pathways $[1,2]$. Hyperhomocysteinemia may be involved in several diseases, such as atherosclerosis, congestive heart failure, osteoporosis, type 2 diabetes, and Alzheimer's disease. Furthermore, evidence shows that high homocysteine levels may accelerate cancer cell growth, although the mechanisms underlying this effect are not known [1-3].

Hyperhomocysteinemia has also been reported in several immune diseases, including human immunodeficiency virus (HIV) infection, common-variable immunodeficiency, systemic lupus erythematosus, rheumatoid arthritis, and inflammatory bowel disease (IBD). Supported by many compelling data, much effort has been expended toward understanding whether hyperhomocysteinemia is an authentic causative and modifiable risk factor for these diseases and toward elucidating the biological mechanisms involved. In immune disorders and diseases, it may influence the immune response by activating and deregulating both innate and adaptive immunity, inducing chemokine and cytokine secretion in monocytes and $\mathrm{T}$ cells, and by supporting $\mathrm{B}$ cell activation [4].

Subjects with folate or vitamin B12 deficiency have increased serum concentrations of homocysteine accompanied by lymphocytosis. These increased homocysteine levels may substantially influence $\mathrm{T}$ cell responses by inhibiting $\mathrm{T}$ cell apoptosis and by supporting $\mathrm{T}$ cell proliferation and secretion of proinflammatory type 1 (IFN- $\gamma$, IL-2, and TNF- $\alpha$ ) and type 17 (IL-17 family) cytokines, but not type 2 cytokines (IL-4 and IL-5). Some of these effects might be mediated by enhancing endoplasmic reticulummitochondrial coupling and increasing mitochondrial respiration [5]. Moreover, Wang et al. showed that relatively 


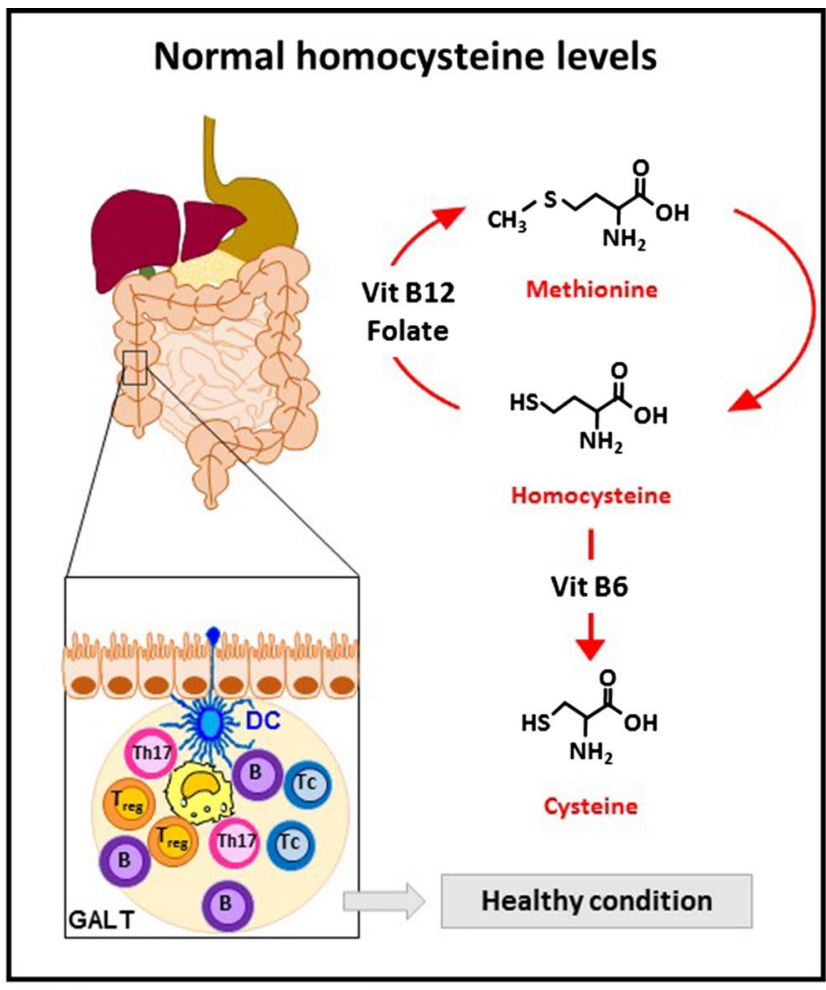

Fig. 1 The left panel depicts methionine metabolism in health. Methionine derived from the diet is converted to homocysteine that is then removed by remethylation to methionine or by transsulfuration to cysteine. Vitamin B12, Vitamin B9 (folate), and Vitamin B6 are essential cofactors for these pathways. Feeding a methionine-enriched

high homocysteine levels upregulate IL-17A expression in T cells via NOP2/Sun RNA Methyltransferase Family Member 2 (NSun2)-mediated RNA methylation, a mechanism also involved in the regulation of cell proliferation and differentiation, cancer development, and endothelial cell activation [6].

Two recent articles from the Chen group located in Wuhan University, China, suggest that homocysteine may affect $\mathrm{T}$ helper $(\mathrm{Th}) 17$ cell differentiation with possible involvement in the development of IBD (including Crohn's disease and ulcerative colitis), chronic relapsing-remitting disorders of unknown etiology, involving a complex interaction of and responses to environmental, genetic, and immune factors. One publication reported that high levels of homocysteine aggravate intestinal inflammation in an animal model of colitis by increasing levels of IL-17 and RAR-related orphan receptor (ROR) $\gamma \mathrm{t}$, the transcription factor driving Th17 cell differentiation [7]. In the second paper, published in this issue of Digestive Diseases and Sciences, Gao et al. investigated the effect of homocysteine in rat intestinal lamina propria lymphocytes in vitro, reporting that cell culture supplementation with homocysteine increases the differentiation of Th17 cells activated through triggering of

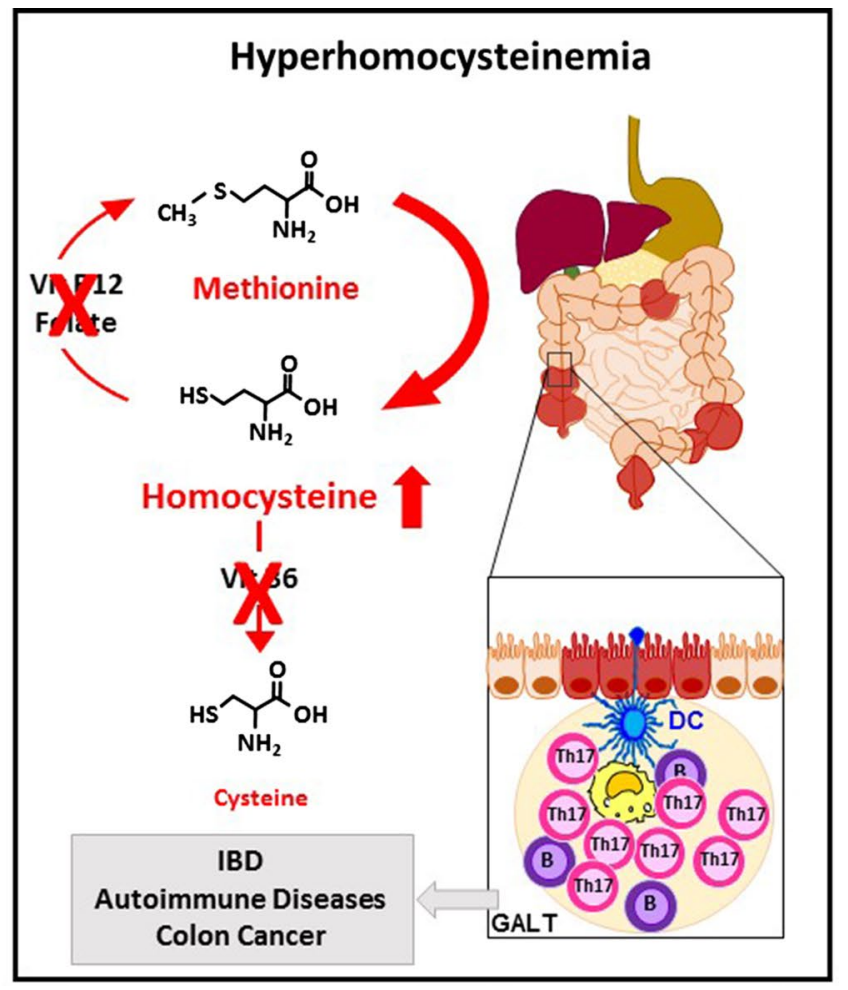

diet or reduction of vitamin B intake causes hyperhomocysteinemia (right panel). High homocysteine levels may induce differentiation of intestinal lamina propria $\mathrm{CD}^{+} \mathrm{T}$ cells into Th17 cells, which are involved in the pathogenesis of IBD, autoimmune disease, and cancer

cluster-of-differentiation (CD)3 and CD28 [8]. The differentiating effect was quite potent since it was detected in cells cultured in the absence of antibodies neutralizing IFN- $\gamma$ and IL-4, which are needed to inhibit Th1 and Th2 cell differentiation and amplify Th17 cell differentiation. Unfortunately, the paper did not investigate the effect of homocysteine on activation of other Th cell subsets, such as Th1, Th2, or on regulatory $\mathrm{T}$ cells. Therefore, the selectivity of the homocysteine effect on Th17 cell activation and differentiation must be inferred only from the medical literature. A second key point that would be interesting to investigate is whether this homocysteine effect is due to the activation of previously differentiated memory/effector Th17 cells, de novo differentiation of naïve $T$ cells, or transdifferentiation of other types of effector Th cells (Th cell plasticity). The importance of these alternatives is that the magnitude of differentiating stimuli may differ among these options and that each option may produce different end effects in vivo. Moreover, further studies should also investigate the contribution of the toxic catabolite HTL which is always present when serum homocysteine is elevated. HTL forms isopeptide bonds with protein lysine residues, altering protein function, forming novel protein epitopes, and activating autoimmune responses 
[2]. Hyperhomocysteinemia, as well as an increased level of homocysteine in the colonic mucosa in IBD patients, decreases intestinal absorption of folate, vitamin B12, and vitamin B6, due to damage of the gut mucosa. This malabsorption is combined with the competitive inhibition of folate absorption by sulfasalazine, a prodrug of 5-aminosalicylic acid commonly used in the treatment of IBD. These events may impair homocysteine metabolism through the remethylation and transsulfuration pathways. An intriguing observation of the report by Gao et al. is that the addition of folic acid to lamina propria cell cultures inhibited Th17 cell differentiation and IL-17 production. These data suggest that dietary supplementation with folic acid may improve IBD progression, in line with the observation that cell culture supplementation with folic acid inhibits the enhancing effect of homocysteine on the growth of colon cancer cell lines in vitro [9]. Moreover, epidemiological data indicate that low dietary folate intake is associated with a higher risk of colorectal cancer in the overall population [9], and a meta-analysis involving 4517 IBD patients reported an overall protective effect of folic acid supplementation on the development of colorectal cancer. This observation is particularly relevant for IBD also since patients with IBD and high homocysteine levels have an increased risk of colorectal cancer development. [10].

Although these data are intriguing, caution must be taken prior to translating these experimental findings into clinical practice. The intestinal epithelium and the immune system are two of the most dynamic sites of cell turnover in mammals. Alterations in the subtle balance of their relationship may have unexpected and disappointing effects, in particular when food-derived agents are prescribed in pharmaceutical doses, as was the case for vitamin E supplementation used to reduce oxidative stress [3]. Folate supplementation can either prevent or exacerbate intestinal tumorigenesis [3], depending on timing and dose of folate intervention, existing risk factors, exposure time to the nutritional deficiency, genetic predisposition, folate receptor (an intriguing tumor-associated antigen) expression, and gut microbiota composition.

Considering future perspectives, the folate form selected for pilot interventions should be carefully evaluated since folic acid, folinic acid, or 5-methyltetrahydrofolate (5-MTHF) may all be used as supplements. 5-MTHF is naturally present in foods and may be preferred to synthetic folic acid since its bioavailability is not affected by methylenetetrahydrofolate reductase polymorphisms, it is absorbed in a broader range of gastrointestinal $\mathrm{pH}$, it masks fewer symptoms of vitamin B12 deficiency, it displays fewer interactions with drugs inhibiting dihydrofolate reductase, and it is devoid of the risks due to the activity of unconverted folic acid in the peripheral blood. Methionine diet restriction could be another strategy to be considered [1-3].
In conclusion, hyperhomocysteinemia due to vitamin B malabsorption may support the proinflammatory activity of Th17 cells in the gut mucosa in IBD, worsening the disease. Therefore, folate might be suggested as a cheap, safe, and well-tolerated dietary supplement that may decrease disease activity. Further experimental studies are needed to investigate which molecule, dietary regimen, dose, and timing may be used to ameliorate the pathologic effects observed in different disease models. Furthermore, IBD is associated with an increased risk of developing autoimmune disease [11], which too might be influenced by hyperhomocysteinemia and, therefore, may benefit from the correction of hyperhomocysteinemia.

\section{Compliance with ethical standards}

Conflict of interest The authors declared no financial conflict of interest.

\section{References}

1. Dinavahi R, Falkner B. Relationship of homocysteine with cardiovascular disease and blood pressure. J Clin Hypertens (Greenwich). 2004;6:494-498.

2. Kumar A, Palfrey HA, Pathak R, Kadowitz PJ, Gettys TW, Murthy $\mathrm{SN}$. The metabolism and significance of homocysteine in nutrition and health. Nutr Metab (Lond). 2017;14:78.

3. Potter JD. The failure of cancer chemoprevention. Carcinogenesis. 2014;35:974-982.

4. Fefelova EV, Tereshkov PP, Dutov AA, Tsybikov NN. Lymphocyte subpopulations and cytokine levels in experimental hyperhomocysteinemia. Bull Exp Biol Med. 2015;159:358-360.

5. Feng J, Lu S, Ding Y, Zheng M, Wang X. Homocysteine activates $T$ cells by enhancing endoplasmic reticulum-mitochondria coupling and increasing mitochondrial respiration. Protein Cell. 2016;7:391-402.

6. Wang N, Tang H, Wang X, et al. Homocysteine upregulates interleukin-17A expression via NSun2-mediated RNA methylation in T lymphocytes. Biochem Biophys Res Commun. 2017;493:94-99.

7. Zhu S, Li J, Bing Y, et al. Diet-induced hyperhomocysteinaemia increases intestinal inflammation in an animal model of colitis. $J$ Crohns Colitis. 2015;9:708-719.

8. Gao X, Li J, Chen M. Effect of Homocysteine on the Differentiation of CD4(+) T Cells into Th17 Cells. Dig Dis Sci. (Epub ahead of print). https://doi.org/10.1007/s10620-018-5177-2.

9. Sanderson P, Stone E, Kim YI, et al. Folate and colo-rectal cancer risk. Br J Nutr. 2007;98:1299-1304.

10. Burr NE, Hull MA, Subramanian V. Folic acid supplementation may reduce colorectal cancer risk in patients with inflammatory bowel disease: a systematic review and meta-analysis. J Clin Gastroenterol. 2017;51:247-253.

11. Halling ML, Kjeldsen J, Knudsen T, Nielsen J, Hansen LK. Patients with inflammatory bowel disease have increased risk of autoimmune and inflammatory diseases. World J Gastroenterol. 2017;23:6137-6146. 